\title{
2-D tilt and 3-D slant illusions in perception and action tasks
}

\author{
Paul B Hibbard \\ School of Psychology, University of St Andrews, St Mary's College, South Street, St Andrews KY16 9JP, \\ Scotland,UK; pbh2@st-andrews.ac.uk

\section{Mark F Bradshaw} \\ Department of Psychology, University of Surrey, Guildford GU2 7XH, UK \\ Received 20 August 2004, in revised form 13 January 2006; published online 25 October 2006
}

\begin{abstract}
There is now a well established dissociation between perception and action based primarily on neuropsychological evidence [Milner and Goodale, 1995 The Visual Brain in Action (Oxford: Oxford University Press)]. Although equivocal, an important source of evidence from normal observers is that 'perceptual illusions' may affect the systems differently. We investigated the relative effects of 2-D tilt and 3-D slant illusions in the two domains, using similar tasks to those employed originally by Milner and Goodale. Subjects were required to either post a card through, or set a paddle to match the orientation of, a plane that was presented in two conditions: surrounded by a striped surface tilted between $+90^{\circ}$ and $-90^{\circ}$ (2-D tilt contrast), or surrounded by a disparity defined surface slanted in depth between $+60^{\circ}$ and $-60^{\circ}$ (3-D depth contrast). For 2-D tilt, action and perception were equally affected by the illusion, whereas in the 3-D condition they were not. Here, the illusion appeared greater in the posting than in the perceptual task. We conclude that, although no qualitative differences exist, there were quantitative differences between perception and action tasks in the binocular condition.
\end{abstract}

\section{Introduction}

The visual system allows us to perceive the world and to act within it. A distinction has been drawn between the visual mechanisms underlying perception and the control of action, and it has been suggested that the two form quasi-independent processing streams projecting to the temporal (ventral stream) and parietal (dorsal stream) lobes of the brain (Milner and Goodale 1995). The major source of evidence for this distinction is from clinical studies, which have demonstrated a double dissociation between some patients (notably RV-Goodale et al 1993) who can perform perceptual tasks but not visually guided behavioural tasks, and others (notably DF-Goodale et al 1991) who perform extremely poorly on perceptual tasks while able to perform visually guided actions well.

Consistent with this evidence, the action-based responses of neurologically intact subjects appear less susceptible to the effects of many common visual illusions where the perception of a target feature is affected by the context within which it is presented. Examples of such illusions used in this type of research include illusory induced motion (Bridgeman et al 1981; Wong and Mack 1981; Dassonville and Bala 2004; Dassonville et al 2004), the Ebbinghaus illusion (Aglioti et al 1995), and the Müller-Lyer illusion (Daprati and Gentilucci 1997; Otto-de Haart et al 1999; Westwood et al 2000). Many other visual illusions might similarly be described as context effects, and therefore also be expected to demonstrate different consequences for perception and the control of action. In the present paper we investigate whether there is a demonstrable dissociation between perception and action responses when subjects are presented with 2-D (tilt) and 3-D (slant) simultaneous-contrast illusions, where the perception of the tilt or slant of a central region is affected by the tilt or slant of its surround (Gibson and Radner 1937; Werner 1937, 1938). 
Experiments that have used visual illusions to dissociate perception and action performance have experienced extensive theoretical and procedural problems (eg Bruno 2001; Carey 2001; Franz 2001; Glover 2002), and have produced many contradictory results. It has been claimed that some experiments may be biased in favour of finding differences in performance as the experimental conditions are often not adequately matched in terms of their design or procedure (see Vishton et al 1999; Franz et al 2000; Mon-Williams and Bull 2000). Also, it has been suggested that the perception and action tasks used in many experiments may be confounded with the types of information required for each task. While many action tasks depend on absolute information to program motor movements to a particular feature, perception tasks often require relative information about different features and so differences in performance may represent informational differences rather than a perception/action distinction per se. To demonstrate the consequences of this, Vishton et al (1999) devised action tasks that required relative information and perceptual tasks that required absolute information and showed that the typical performance differences between perception and action could be reversed.

A related complication, raised by Dyde and Milner (2002), is that finding no difference between the effects of an illusion on perception and action may not necessarily contradict the idea of separate processing streams. This is because a particular illusion may simply occur early enough in visual processing so that it affects both streams. They found that the tilt illusion affected perception and action to an equal degree, whereas the rod-and-frame illusion affected perception but not action. They argued that the tilt illusion occurs early in processing (most likely in cortical area V1), and so will influence both dorsal and ventral processing streams, whereas the rod-and-frame illusion arises much later, in higher level areas of the ventral processing stream.

A final concern is the fact that a simple quantitative difference between the effects of an illusion on two different dependent measures (ie perception and action responses) is difficult to interpret (eg Mack et al 1985; Post and Welch 1996; Smeets and Brenner 1996; Wraga et al 2000; Bradshaw and Watt 2002). A difference in absolute performance may reflect the fact that anatomically distinct control processes are involved, but it may also arise from the fact that the properties of the effector systems themselves differ. For example, perceived size can vary over a large range whereas grip aperture can vary only over a very narrow range. The functions that relate perceived size and grip aperture to physical size therefore may have different slopes and so differences in performance would be expected even if a common representation were used to control perceptual and visuo-motor behaviour (Franz 2003).

In the present paper we used 2-D and 3-D simultaneous-contrast illusions and a posting task similar to that devised by Goodale et al (1994) to illustrate perception/ action dissociations in their seminal work with patient DF. The influence of the 2-D induced-tilt illusion has been reported previously (Dyde and Milner 2002). However, owing to the equivocal nature of many experiments in this area, it is important to review such results, particularly as we employ a technique which allows a direct quantitative, in addition to a qualitative, comparison between the two domains. As introduced above, using the magnitude estimation task, employed by Dyde and Milner (2002) to measure the size of the illusion, is problematic as it is not clear what perceived tilt corresponds to a particular setting. For this reason quantitative comparisons between the results from two domains are difficult. The technique used here, adopted from traditional psychophysical methods, established a 'subjective vertical' (2-D) or 'subjective frontoparallel' (3-D) so that a consistent criterion could be compared across experimental conditions. This technique has been used many times to establish the strength of simultaneous or successive contrast illusions and by determining a 'null point' (subjective vertical/frontoparallel) we avoid the problems of calibration (nonlinearities 
or differences in gain) that are intrinsic to many forms of magnitude estimation as used in previous studies that have contrasted the two domains.

The 2-D tilt illusion also forms a useful comparison for the 3-D contrast illusion, which is of particular interest since it represents a contextual effect on the interpretation of binocular cues. Such cues are often considered to be particularly involved in the control of action tasks as, in principle, they provide accurate metric 3-D information about the world (Servos et al 1992; Dijkerman et al 1996; Jackson et al 1997; Marotta et al 1997; Watt and Bradshaw 2000). The use of binocular cues for the control of action is supported by the fact that binocular depth processing is associated with activity in areas V3A and MT (DeAngelis et al 1998; Backus et al 2001), regions with clear connections with the parietal areas (Lennie 1998). Considerations of the control of action often stress the use of absolute visual information, and therefore the potential for immunity from contextual effects. These two considerations make the 3-D contrast illusion, essentially a contextual illusion in the perception of depth from binocular disparity, a particularly interesting case to consider.

Our interest in this case is independent of considerations of whether this is an effect that occurs early or late in visual processing. Milner and Goodale (1995) provided a compelling computational argument that the processes underlying action and perception performance require qualitatively different transformations of the sense data. That is, action requires absolute information that is updated frequently to take account of self- or object movement, whereas perception requires view-independent, relative information that persists over time. The absolute information required by action is most readily available from cues such as binocular disparity and motion parallax. An illusory effect in the use of binocular disparity would mean that this primary source of absolute spatial information does not, in practice, support a veridical representation of surface structure for the control of action.

\section{Method}

\subsection{Subjects}

Eight right-handed adult volunteers participated in the experiment. All had normal or corrected-to-normal vision and a stereoacuity of at least $40 \mathrm{~s}$ of arc (Randot Stereo Tests).

\subsection{Apparatus}

Stimuli were presented on a 19-inch Flatscreen ViewSonic monitor which was viewed via a mirror set at $45^{\circ}$ to the midline. The subjects perceived a single virtual stimulus immediately in front of them. Experiments were performed in the dark, and the monitor surround and other objects were not visible. A vertical board was positioned in front of the subject, at the same distance as the reflected image of the monitor, to provide haptic feedback. This board was positioned at right angles to the subject's midline (see figure 1). The subject's head was restrained by a head-and-chin rest. The viewing distance was $30 \mathrm{~cm}$. Dichoptic presentation was achieved with the use of Stereographics Crystaleyes liquid-crystal shutter glasses. The monitor refresh rate was $120 \mathrm{~Hz}$. Crosstalk between the left and right eye images was minimised $(<1 \%)$ by using only the red gun of the monitor. The orientation of an illuminated posting card was monitored with the Minibird real-time electromagnetic tracking system (Ascension Technology).

\subsection{Stimuli}

Stimuli consisted of a circular disc (30 deg diameter). In the 2-D task the disc was

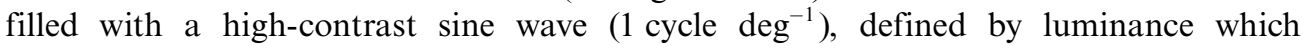
could be oriented at various angles. The phase was varied randomly between trials. In the 3-D task, the disc was filled with small 'blobs' $\left(15\right.$ dots $\left.\mathrm{deg}^{-2}\right)$ which were defined by a Gaussian luminance profile with a standard deviation of $1 \mathrm{~mm}$. The slant of the 


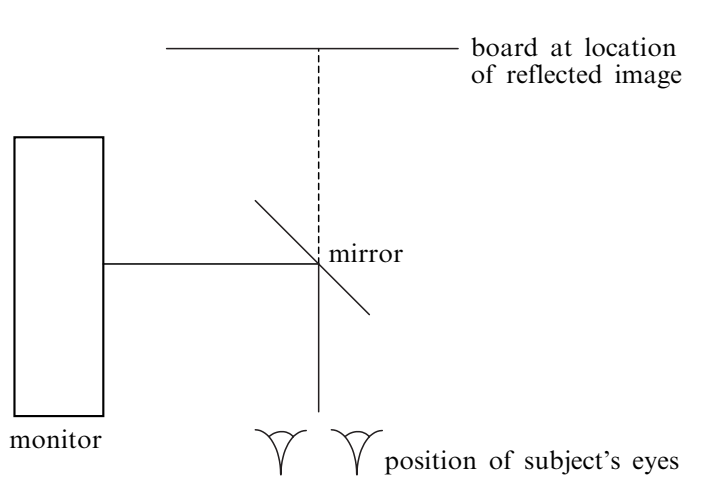

Figure 1. The experimental setup. The monitor was viewed through a mirror positioned at $45^{\circ}$ to the subject's cyclopean line of sight. A board was positioned directly in front of the subject, at the same distance as the reflected image of the monitor, to provide haptic feedback.

surface was defined by the binocular disparity of these blobs; texture and perspective information were not varied across conditions, and were thus consistent with a frontoparallel surface. Binocular disparities were depicted with subpixel accuracy. A central $7.5 \mathrm{deg}$ disc in both cases defined the target area. The orientation of this area could be changed independently from the background disc according to the demands of the particular experimental condition (see below). In the 3-D task, the central disc contained a small gap (1 deg wide) which formed a 'slot' for the posting task; in the 2-D task, the central 'dark bar' of the sine wave performed the same role.

\subsection{Perceptual tasks}

On each trial subjects adjusted the orientation of the central disc until (i) its stripes appeared vertical (2-D task) or (ii) its surface appeared frontoparallel (3-D task). The orientation of the 2-D stripes on, or 3-D slant of, the flanking disc slanted about a vertical axis with a slant of either $0^{\circ}, \pm 15^{\circ}, \pm 30^{\circ}, \pm 45^{\circ}$, or $\pm 60^{\circ}$. Each flanking orientation was presented four times in the 2-D and 3-D conditions, creating 40 trials in total in both conditions. The value that created the maximum illusion was selected for use in the action-based tasks.

\subsection{Action tasks}

Subjects were requested to post an initially horizontally hand-held posting card through the central black area (slot) of the central disc. In the 2-D tilt conditions the orientation of the stripes in the flanking surface was set at either $0^{\circ}$ or $\pm 15^{\circ}$. The orientation of the stripes on the central surface was either $0^{\circ}, \pm 3^{\circ}$, or $\pm 6^{\circ}$. This allowed us to determine the point of 'subjective vertical' for the posting task, allowing for a direct comparison with the perceptual results (this is described in section 3 below). In the 3-D slant conditions the orientation of the flanking surface was set at either $0^{\circ}$ or $\pm 30^{\circ}$ and the slant of the central surface was either $0^{\circ}, \pm 6^{\circ}$, or $\pm 12^{\circ}$; the slant of the central slot (as defined by binocular disparity) was equal to the slant of the central surface. Each combination of surround and target orientation was presented four times, creating a total of 60 trials in each condition. Any intrinsic bias in perceived vertical or frontoparallel (ie manifest without flanking stimulus) was corrected by subtracting the responses to the central disc when presented in isolation.

\section{Results}

\section{$3.12-D$ tilt}

The perceptual results for the 2-D induced tilt condition are shown in figure 2. Figure $2 \mathrm{a}$ shows the degree of tilt set by observers to counteract the tilt induced by the surround. In agreement with previous studies (Clifford et al 2000), a surround orientation of $\pm 15^{\circ}$ induced the greatest tilt. At this orientation, a tilt of approximately $3^{\circ}$ was induced.

For the action task, surround tilts of $0^{\circ}$ and $\pm 15^{\circ}$ were selected. The final orientation of the card, as a function of the orientation of both the target and the surround, 


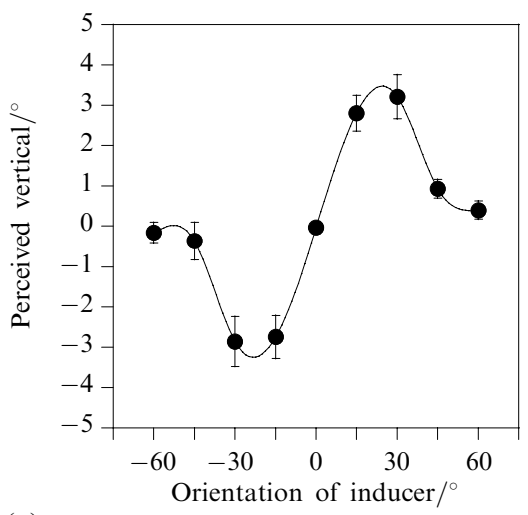

(a)

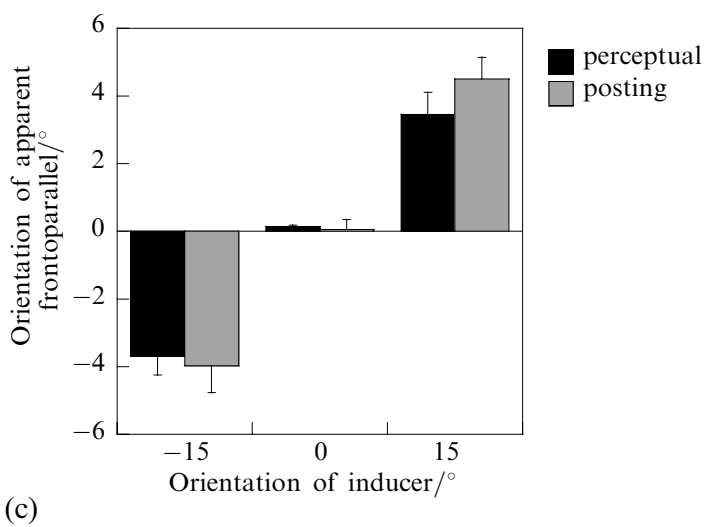

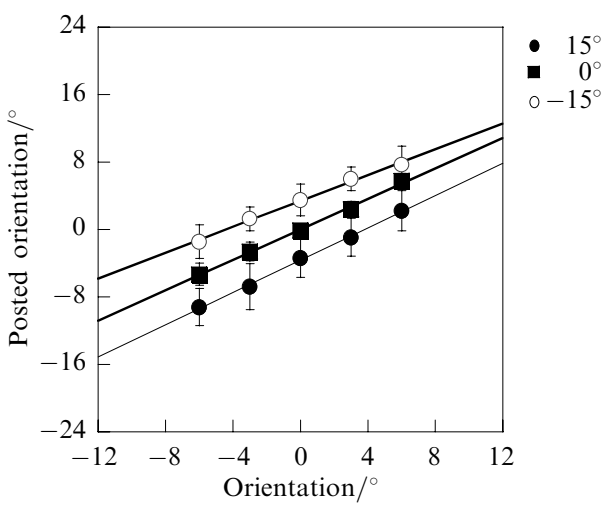

(b)

Orientation $/^{\circ}$

Figure 2. Results for the 2-D induced tilt condition. (a) Perceptual results, showing the orientation of the target that was perceived as vertical, as a function of the orientation of the inducer. (b) Posting results, showing the final orientation of the card as a function of the orientation of the target. Results are plotted separately for the three different orientations of the inducer, which had a clear effect on performance. (c) Comparison of the magnitude of the effect between the perceptual and posting conditions. In all cases error bars represent \pm 1 SEM.

is shown in figure $2 b$. These results show that subjects' posting movements successfully tracked the orientation of the target - in each case the graph shows a slope of close to one, and the intercept is close to zero when the surround orientation is zero. The induced tilt is evident in these results by the vertical shift between the conditions. In order to compare this effect quantitatively with the perceptual results, a 'subjective vertical' was calculated in each case. This represents the physical orientation that the target would need to be set to, in order for the card to be posted with a vertical orientation. These results are plotted in figure $2 \mathrm{c}$ for direct comparison with the perceptual results. It is evident from this graph that the effect of the induced tilt on perception and action is similar both qualitatively and quantitatively. This was confirmed by an analysis of variance, which found no effect of condition (perception versus action) $\left(F_{1,7}=1.019, \mathrm{~ns}\right)$, a significant effect of surround tilt $\left(F_{2,14}=41.9, p<0.001\right)$, and no significant interaction $\left(F_{2,14}=2.701, \mathrm{~ns}\right)$.

\subsection{3-D slant}

Results for the 3-D slant condition are presented in a similar manner in figure 3 . Figure $3 \mathrm{a}$ shows the perceptual matching results. Here, the greatest slant was induced by a surround slant of $\pm 30^{\circ}$. Therefore, in the posting task, inducing slants of $0^{\circ}$ and $\pm 30^{\circ}$ were used. The results of the posting task are shown in figure $3 \mathrm{~b}$. Again, posting orientation accurately reflected surface orientation when the inducing slant was zero. 


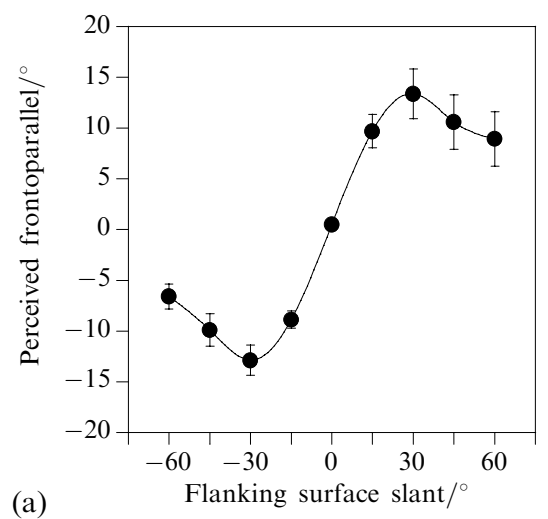

(a)

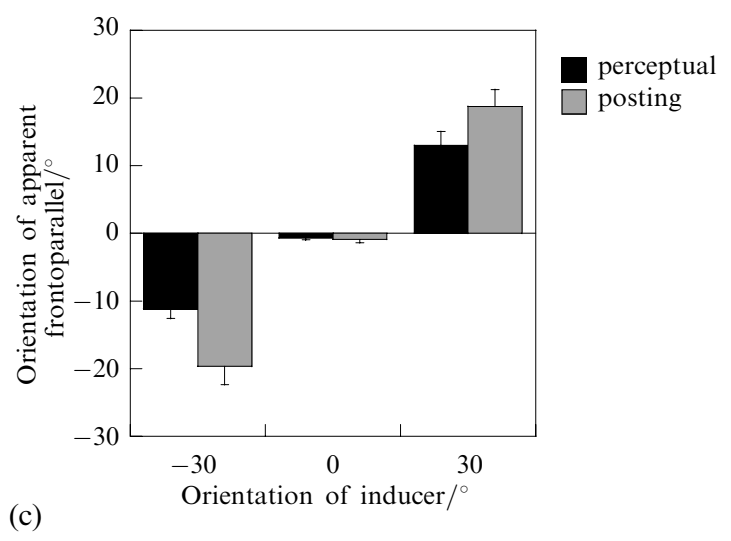

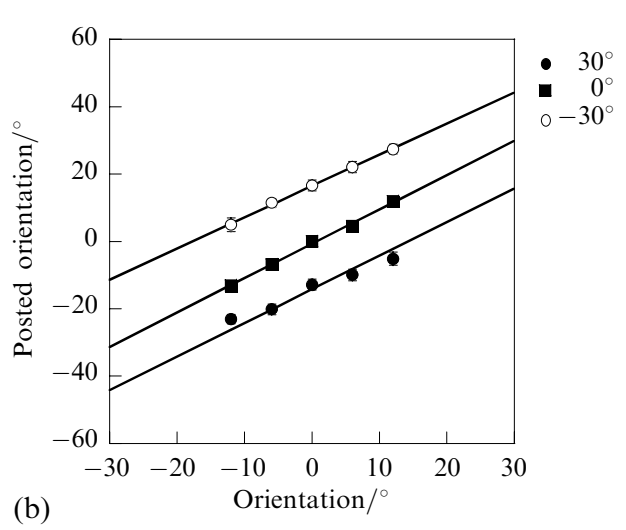

(b)

perceptua

(1)

Figure 3. Results for the 3-D induced slant condition. (a) Perceptual results, showing the orientation of the target that was perceived as frontoparallel, as a function of the orientation of the inducer. (b) Posting results, showing the final orientation of the card as a function of the orientation of the target. Results are plotted separately for the three different orientations of the inducer, which had a clear effect on performance. (c) Comparison of the magnitude of the effect between the perceptual and posting conditions.

That is, as the slant of the central surface was varied away from frontoparallel, subjects varied the orientation at which they posted the card through the slot in a consistent manner. The results show a clear induced slant in the other two conditions, as indicated by the vertical shifts between the three sets of data. A 'subjective frontoparallel' was estimated in each case, ie the inducing slant that leads to a posting slant of $0^{\circ}$. These values are plotted in figure $3 \mathrm{c}$, together with the perceptual data.

It should be noted that, to obtain these values from figure $3 \mathrm{~b}$, the regression lines were extrapolated beyond the range of the data points in both cases. This is because the size of the effect was greater than anticipated, as can be seen from the fact that in neither case was a posting orientation of $0^{\circ}$ observed. While this extrapolation should be borne in mind, it appears to be justified since the slope of the curves is very close to 1 across the whole range of posting responses, from $-23^{\circ}$ to $27^{\circ}$.

Again, the effect of the illusion on the posting response mirrors the perceptual effect, at least qualitatively. However, the magnitude of the effect on posting is approximately twice that of the perceptual effect. This is confirmed by an analysis of variance, which found no effect of condition $\left(F_{1,7}=0.358, \mathrm{~ns}\right)$, a significant effect of surround slant $\left(F_{2,14}=84.22, p<0.001\right)$, and a significant interaction $\left(F_{2,14}=11.865, p<0.005\right)$. In other words, the effective frontoparallel was affected by the slant of the surround, and this effect was greater for action than for perception. 


\section{Discussion}

The present study was designed to compare the relative size of the 2-D tilt and 3-D slant contrast illusions on the putative perception and action subsystems (Milner and Goodale 1995). The effect of the illusions was clearly manifest in both the perception and action conditions. For the 2-D illusion, the magnitude of the effect was similar for perception and action, consistent with the results reported by Dyde and Milner (2002); and for the 3-D illusion, the magnitude of the effect on the action response was significantly greater than its effect on the perception response.

It is not clear why a greater illusory effect on action than on perception was observed. It is interesting to note that Knill (2005) recently reported that, in a cue perturbation experiment, action tasks depended on binocular cues to a greater extent than did perceptual tasks. This result may best be interpreted in terms of possible differences in the manner in which the information provided by visual cues is put to use in these different tasks, rather than necessarily in terms of differences in the underlying processing of this information in two independent processing streams.

As discussed in section 1, Dyde and Milner (2002) have argued that the failure to find an illusory effect on action does not contradict the idea of separate processing streams, as such an effect might arise early in processing. Our prime motivation in the use of the 3-D illusion was that binocular cues are often considered to be particularly involved in the control of action tasks as, in principle, they provide accurate metric 3-D information about the world. Therefore, we reasoned, they should be particularly immune to the effects of visual illusions. However, given Dyde and Milner's argument, the use of the 3-D illusion may also be a useful test because, although V1 is involved in the computation of binocular disparity (eg Cumming and Parker 1997), the recovery of depth information occurs at a later stage in processing. Cortical areas that are considered to be in both the ventral and dorsal streams are involved in these latter stages of the processing of binocular information (Neri 2005). Neri suggested that processing in the ventral and dorsal streams may support perception and action, respectively, although importantly defines action in terms of the control of vergence eye movements. It is not clear whether the representation of surface layout required for the control of manual actions such as those studied here would depend on processing in dorsal or ventral areas. Indeed, the question of what constitutes the class of actions coming under dorsal-stream control is by no means clear-cut at present (Gonzalez et al 2005). However, the fact that we find performance to be affected by an illusion in the processing of binocular disparity is interesting, given its important role in the visual control of prehension. Consistent with these results, we have also established that prehension is subject to similar biases in the recovery of shape and distance from binocular information as are well known in the perceptual domain (Johnston 1991; Bradshaw and Hibbard 2003; Hibbard and Bradshaw 2003).

The theoretical and empirical problems experienced through the use of the visualillusion paradigm in dissociating perception and action systems suggests that it may, at best, be problematic in providing a robust, unambiguous test of the underlying questions. The fact that, in the present paper, the predicted difference was not found between perception and action responses to 2-D tilt and 3-D slant illusions suggests that the systems are not dissociable, at least in terms of the representations of surfaces and objects that are relied on by the two systems. As argued in section 1, contrasting the two illusions as 'early' and 'late' does not affect this conclusion. To appreciate fully the implications of the potential immunity of action planning from visual illusions for processing in the dorsal and ventral streams, it is important to consider which aspects of processing are common to both streams and which are independent. The current results suggest that a substantial core of the processing underlying the representation of the 3-D shape and layout of surfaces is common to both streams. 
Acknowledgments. This research was supported by the Wellcome Trust. Thanks to Rob van der Willigen, Simon Watt, and Terry Thacker for their help in the preparation of this manuscript.

\section{References}

Aglioti S, Goodale M A, DeSouza J F X, 1995 "Size-contrast illusions deceive the eye but not the hand" Current Biology 5 679-685

Backus B T, Fleet D J, Parker A J, Heeger D J, 2001 "Human cortical activity correlates with stereoscopic depth perception" Journal of Neurophysiology 86 2054-2068

Bradley D C, Chang G C, Andersen R A, 1998 "Encoding of three-dimensional structure-frommotion by primate area MT neurons" Nature $392714-717$

Bradshaw M F, Hibbard P B, 2003 "Reaching for virtual objects: binocular disparity, retinal motion and the control of prehension" Arquivos Brasileiros de Oftalmologia 66 53-61

Bradshaw M F, Watt S J, 2002 "A dissociation of perception and action in normal human observers: the effect of temporal-delay" Neuropsychologia $401766-1778$

Bridgeman B, Kirch M, Sperling A, 1981 "Segregation of cognitive and motor aspects of visual function using induced motion" Perception \& Psychophysics 29 336-342

Bruno N, 2001 "When does action resist visual illusions?" Trends in Cognitive Sciences 5 379-382

Carey D P, 2001 "Do action systems resist visual illusions?" Trends in Cognitive Sciences 5 109-113

Clifford C W G, Wenderoth P, Spehar B, 2000 "A functional angle on some aftereffects in cortical vision" Proceedings of the Royal Society of London B $2671705-1710$

Cumming B G, 2002 "Stereopsis: where depth is seen" Current Biology 12 R93-R95

Cumming B G, Parker A J, 1997 "Responses of primary visual cortical neurons to binocular disparity without depth perception" Nature $389280-283$

Daprati E, Gentilucci M, 1997 "Grasping an illusion” Neuropsychologia $351577-1582$

Dassonville P, Bala J K, 2004 "Are the original Roelofs effect and the induced Roelofs effect confounded by the same expansion of remembered space?" Vision Research 44 1025-1029

Dassonville P, Bridgeman B, Bala J K, Thiem P, Sampanes A, 2004 "The induced Roelofs effect: two visual systems or the shift of a single reference frame?" Vision Research 44 603-611

DeAngelis G C, Cumming B G, Newsome W T, 1998 "Cortical area MT and the perception of stereoscopic depth" Nature $394677-680$

Dijkerman H C, Milner A D, Carey D P, 1996 "The perception and prehension of objects oriented in the depth plane. I. Effects of visual form agnosia" Experimental Brain Research $112442-451$

Dodd J V, Krug K, Cumming B G, Parker A J, 2001 "Perceptually bistable three-dimensional figures evoke high choice probabilities in cortical area MT" Journal of Neuroscience 21 $4809-4821$

Dyde R T, Milner A D, 2002 "Two illusions of perceived orientation: one fools all of the people some of the time, but the other fools all of the people all of the time" Experimental Brain Research $144518-527$

Franz V H, 2001 "Action does not resist visual illusions" Trends in Cognitive Sciences 5 457-459

Franz V H, 2003 "Manual size estimation: a neuropsychological measure of perception?" Experimental Brain Research $151471-477$

Franz V H, Gegenfurtner K R, Bülthoff H H, Fahle M, 2000 "Grasping visual illusions: no evidence for a dissociation between perception and action" Psychological Science $1120-25$

Gibson J J, Radner M, 1937 "Adaptation, after-effect, and contrast in the perception of tilted lines. I. Quantitative studies" Journal of Experimental Psychology 20453 - 467

Glover S, 2002 "Visual illusions affect planning but not control" Trends in Cognitive Sciences 6 $288-292$

Gonzalez C L R, Ganel T, Goodale M A, 2005 "Perceptual illusions affect visually-guided actions with the non-dominant but not with the dominant hand" Journal of Vision 5 354a (abstract)

Goodale M A, Jakobson L S, Milner A D, Perrett D I, Benson P J, Hietanen J K, 1994 "The nature and limits of orientation and pattern processing supporting visuomotor control in a visual form agnosic" Journal of Cognitive Neuroscience 6 46-56

Goodale M A, Milner A D, Jakobson L S, Carey D P, 1991 "A neurological dissociation between perceiving objects and grasping them" Nature $349154-156$

Goodale M A, Murphy K J, Meenan J-P, Racicot C I, Nicholle D A, 1993 "Spared object perception but poor object-calibrated grasping in a patient with optic ataxia" Society of Neuroscience Abstracts 18775 (abstract)

Hibbard P B, Bradshaw M F, 2003 "Reaching for virtual objects: binocular disparity and the control of prehension" Experimental Brain Research 148 196-201 
Jackson S R, Jones C A, Newport R, Pritchard C, 1997 "A kinematic analysis of goal-directed prehension movements executed under binocular, monocular and memory-guided viewing conditions" Visual Cognition 4113 - 142

Johnston E B, 1991 "Systematic distortions of shape from stereopsis" Vision Research 311351 - 1360

Knill D C, 2005 "Reaching for visual cues to depth: The brain combines depth cues differently for motor control and perception" Journal of Vision $5103-115$

Lennie P, 1998 "Single units and visual cortical organization" Perception 27 889-935

Mack A, Fendrich R, Chambers D, Heuer F, 1985 "Perceived position and saccadic eye-movements" Vision Research $25501-505$

Marotta J J, Behrmann M, Goodale M A, 1997 "The removal of binocular cues disrupts the calibration of grasping in patients with visual form agnosia" Experimental Brain Research 116 $113-121$

Milner A D, Goodale M A, 1995 The Visual Brain in Action (Oxford: Oxford University Press)

Mon-Williams M, Bull R, 2000 "The Judd illusion: evidence for two visual streams or two experimental conditions?" Experimental Brain Research $130273-276$

Neri P, 2005 "A stereoscopic look at visual cortex" Journal of Neurophysiology 93 1823-1826

Otto-de Haart E G, Carey D P, Milne A B, 1999 "More thoughts on perceiving and grasping the Müller-Lyer illusion" Neuropsychologia 37 1437-1444

Post R B, Welch R B, 1996 "Is there dissociation of perceptual and motor responses to figural illusions?" Perception 25569 - 581

Servos P, Goodale M A, Jakobson L S, 1992 "The role of binocular vision in prehension: a kinematic analysis" Vision Research $321513-1521$

Smeets J B J, Brenner E, 1995 "Perception and action are based on the same visual information: distinction between position and velocity" Journal of Experimental Psychology 21 19-31

Vishton P M, Rea J G, Cutting J E, Nuñez L N, 1999 "Comparing effect of the horizontal - vertical illusion on grip scaling and judgment: relative versus absolute, not perception versus action" Journal of Experimental Psychology: Human Perception and Performance 25 1659-1672

Watt S J, Bradshaw M F, 2002 "Binocular cues are important in controlling the grasp but not the reach in natural prehension movements" Neuropsychologia 381473 - 1481

Werner H, 1937 "Dynamics in binocular depth perception" Psychological Monographs 49 1-120

Werner H, 1938 "Binocular depth contrast and the conditions of the binocular field" American Journal of Psychology $\mathbf{5 1} 489-497$

Westwood D A, Heath M, Roy E A, 2000 "The effect of a pictorial illusion on closed-loop and open-loop prehension" Experimental Brain Research 134456 -463

Willigen R F van der, Bradshaw M F, Hibbard P B, 2000 "2-D tilt and 3-D slant illusions in perception and action tasks" Perception 29 Supplement, 52 (abstract)

Wong E, Mack A, 1981 "Saccadic programming and perceived location" Acta Psychologica 48 $123-131$

Wraga M, Creem S H, Proffitt D R, 2000 "Perception-action dissociations of a walkable MüllerLyer configuration" Psychological Science 11 239-243 


\section{PERCEPTION}

VOLUME 352006

www.perceptionweb.com

Conditions of use. This article may be downloaded from the Perception website for personal research by members of subscribing organisations. Authors are entitled to distribute their own article (in printed form or by e-mail) to up to 50 people. This PDF may not be placed on any website (or other online distribution system) without permission of the publisher. 